\title{
ALTERAÇÃO DA ATIVIDADE ENZIMÁTICA EM ORGANISMOS AQUÁTICOS POR POLUENTES DE ORIGEM AGRÍCOLA: UMA ABORDAGEM GERAL E SOBRE A SUSCETIBILIDADE DA FOSFATASE ÁCIDA
}

\author{
Claudio Martín Jonsson* \\ Embrapa Meio Ambiente, Rod. SP 340 - km 127,5, 13820-000 Jaguariúna - SP, Brasil \\ Hiroshi Aoyama \\ Departamento de Bioquímica, Instituto de Biologia, Universidade Estadual de Campinas, CP 6109, 13083-970 Campinas - SP, Brasil
}

Recebido em 26/2/09; aceito em 29/10/09; publicado na web em 12/3/10

\begin{abstract}
ALTERATION OF ENZYMATIC ACTIVITY IN AQUATIC ORGANISMS BY AGRICULTURAL POLLUTANTS: A GENERAL APPROACH AND THE SUSCEPTIBILITY OF THE ACID PHOSPHATASE. The input of agrochemicals in the aquatic compartment can results in biochemical injuries for living organisms. In this context, the knowledge of alterations of enzymatic activities due the presence of agriculture pollutants contributes for the elucidation of the mechanisms of toxicity, implementation of economic methods for monitoring purposes and establishment of maximum allowed concentrations. In the present work, the above considerations are discussed, and data concerning changes in enzymatic function by pesticides and fertilizer contaminants are reviewed. Also, we focused on the acid phosphatase due its susceptibility to several pollutants and diversity in cellular functions.
\end{abstract}

Keywords: enzyme; biomarker; pollution.

\section{INTRODUÇÃO}

Com a utilização de modernas práticas agrícolas, que resultaram na maior produção de alimentos, intensificaram-se os problemas causados por pragas. Além dos ácaros, nematoides, fungos e plantas daninhas, eram conhecidas cerca de 68.000 espécies de insetos de ação prejudicial até finais do século $\mathrm{XX}$. ${ }^{1} \mathrm{O}$ combate a estes organismos se faz através do uso de produtos químicos denominados agrotóxicos, sendo que sua utilização abusiva tem ocasionado problemas ao Homem e ao meio ambiente, além de representar uma constante ameaça para as gerações futuras e o equilíbrio dos ecossistemas. Entre estes compostos estão incluídos os inseticidas, acaricidas, herbicidas, fungicidas, nematicidas, entre outros.

Os efeitos desses compostos para os organismos aquáticos são muito variados, seja pela quantidade de ingredientes ativos presentes no mercado, que são aproximadamente 300, no Brasil, seja pela enorme gama de espécies existentes nos corpos d'água. ${ }^{2,3}$ Assim sendo, a toxicidade das moléculas para uma determinada espécie pode variar até mais de 25.000 vezes e apresentar efeito letal na ordem de alguns microgramas por litro. ${ }^{4,5}$

Lodo de esgoto é uma denominação genérica para o resíduo sólido gerado pelos sistemas de tratamento de águas residuais. Trata-se de um material heterogêneo, cuja composição depende do tipo de tratamento empregado para purificar o esgoto e das características das fontes geradoras (população e indústrias). ${ }^{6} \mathrm{O}$ uso de lodo de esgoto como fonte alternativa de nutrientes (fertilizante) para as plantas tem sido apresentado como uma opção para seu descarte devido ao seu alto teor de matéria orgânica. A principal fonte desses elementos metálicos é o setor industrial. Deve-se levar que consideração que a preocupação com metais pesados e os possíveis efeitos prejudiciais a eles associados foi acentuada a partir do uso do lodo de esgoto em solos agrícolas. ${ }^{7}$ Entretanto, a presença desses metais não é exclusividade destes resíduos, uma vez que fertilizantes químicos, corretivos e defensivos podem também contê-los. ${ }^{8}$ Além de metais, o lodo de esgoto pode também conter contaminantes orgânicos como naftalenos e LAS (alquil benzeno linear sulfonado). ${ }^{9}$

\footnotetext{
*e-mail: jonsson@cnpma.embrapa.br
}

Considerando-se o compartimento aquático como um dos receptáculos finais de poluentes químicos, tanto de origem agrícola como urbana, estes ingressam através de diferentes vias: escoamento superficial em áreas urbanas e rurais; ${ }^{10,11}$ deposição atmosférica; ${ }^{12,13}$ percolação através do solo previamente contaminado; ${ }^{14,15}$ descargas acidentais; ${ }^{16,17}$ lavagem de equipamentos de aplicação e manuseio inadequado de embalagens para descarte. ${ }^{18,19}$ Consequentemente, os efeitos adversos da interação poluente químico - biota é sentido nos diferentes níveis tróficos da cadeia alimentar em um sistema aquático, ${ }^{20,21}$ sendo as alterações bioquímicas uma das manifestações que podem indicar o impacto dos contaminantes no compartimento ambiental.

Dentre os componentes bioquímicos, as enzimas constituem um grupo de substâncias, comumente proteínas, com função catalisadora nas vias metabólicas, podendo tal função ser alterada na presença de um xenobiótico de origem agrícola. Na Figura 1 são mostrados os mecanismos de reação de uma enzima (E), na ausência (reações na horizontal) e na presença de um inibidor (I) (reações na vertical). A enzima (E) reage com o substrato (S), para formar o complexo enzima-substrato (ES) e, posteriormente, liberar o produto $(\mathrm{P})$. A formação do complexo enzima-substrato é regida pela constante de Michaelis (Km). Entretanto, a reação catalisada pela enzima pode ser alterada, por exemplo, na presença de um agente químico com ação inibitória (I). Este inibidor (I) pode se ligar à enzima livre (E), atuando como um inibidor do tipo competitivo (Figura 1A), formando um complexo enzima-inibidor (EI) inativo, cuja formação é regida pela constante de inibição (Ki). Um inibidor (I) pode, também, se ligar à enzima livre (E) ou ao complexo enzima-substrato (ES) formando, no final, o complexo enzima-substrato-inibidor (ESI) inativo, atuando, neste caso, como um inibidor do tipo não competitivo (Figura 1B). As reações de formação do complexo EI e do complexo ESI são regidas pelas constantes de inibição Ki e Ki', respectivamente.

Na presente revisão são abordados os efeitos de diversos contaminantes de origem agrícola, tais como agroquímicos e resíduos presentes nos lodos usados com fertilizantes, sobre sistemas enzimáticos de organismos aquáticos. Uma abordagem especial será dada à fosfatase ácida, devido a sua susceptibilidade a poluentes e diversificação de funções no metabolismo celular. 


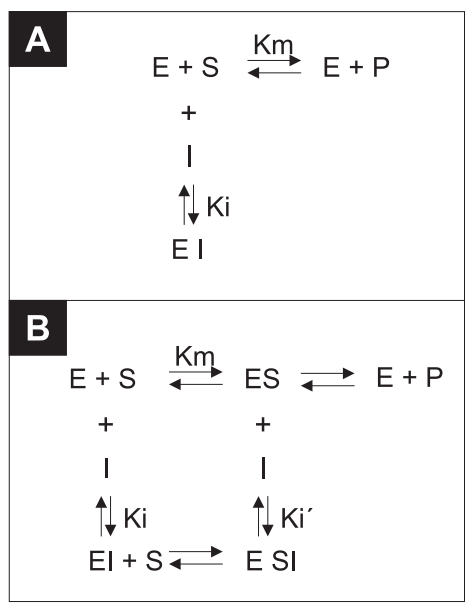

Figura 1. Mecanismos de reação de uma enzima alterados pela presença de inibidores. A) Na presença de um inibidor competitivo. B) Na presença de um inibidor não- competitivo

\section{ALTERAÇÕES BIOQUÍMICAS COMO INDICADORES DE EFEITOS DE POLUENTES NO COMPARTIMENTO AQUÁTICO}

O conceito básico que sustenta a utilização de bioindicadores de poluição ambiental se baseia no fato que os distúrbios por xenobióticos no meio ambiente levam, inicialmente, a uma perturbação de uma reação bioquímica em um determinado organismo. ${ }^{22}$ Se estas alterações bioquímicas forem observadas com uma certa antecedência, pode ser possível a identificação de problemas ambientais antes que o ecossistema aquático, como um todo, seja afetado..$^{23,24}$

Por causa destas características, os biomarcadores ao nível bioquímico são apontados como sistemas de "sinal de alerta" na avaliação da saúde ambiental. ${ }^{25}$

Durante a exposição a vários tipos de contaminantes, os mesmos se concentram em células e tecidos da comunidade aquática podendo promover a alteração de sistemas enzimáticos responsáveis por processos vitais, ocorrendo: a) aumento da atividade enzimática no meio extracelular por extravasamento da proteína para este meio, ${ }^{26,27}$ b) aumento da atividade enzimática no meio extracelular ou intracelular por ativação enzimática, através da interação direta do agente químico com a enzima; ${ }^{28,29} \mathrm{c}$ ) aumento da atividade enzimática intracelular por indução na síntese da proteína, ${ }^{28,30} \mathrm{~d}$ ) diminuição da atividade no meio extracelular ou intracelular por inibição, através da interação direta do agente químico com a proteína; ${ }^{31,32}$ e) diminuição da atividade no meio intracelular por comprometimento da membrana celular e extravasamento da proteína. ${ }^{33}$

A ação de poluentes tem sido estudada nas atividades de enzimas de diversas vias metabólicas, tais como, via glicolítica; ${ }^{34,35}$ via da pentose-fosfato; ${ }^{36,37}$ ciclo de Krebs; $;^{38,39}$ síntese de aminoácidos; ${ }^{35,39}$ degradação de proteínas; ${ }^{40,41}$ cadeia respiratória; ${ }^{38,42,43}$ síntese de ácidos graxos ${ }^{44}$ metabolismo dos lipídeos; ${ }^{41,45}$ metabolismo do nitrogênio; ${ }^{46,47}$ e fotossíntese. ${ }^{48,49}$

Nos últimos anos, importância especial tem sido dada aos efeitos sobre enzimas envolvidas no processo de biotransformação de xenobióticos, tanto da Fase I como da Fase II. Entre as primeiras, associadas às atividades do citocromo $\mathrm{P} 450$ e de monoxigenases de função mista (MFO), têm sido avaliadas a etoxiresorufina-o-deetilase (EROD) e benzopireno monoxigenases.$^{50-52}$ Com relação às enzimas de conjugação na Fase II, a glutationa-S-transferase (GST) e a UDP-glucuronosiltransferase (UDPGT) têm sido também bastante estudadas em organismos expostos em condições de laboratório e nos coletados de áreas impactadas. ${ }^{35,53,54}$
As inibições das atividades da acetilcolinesterase e delta-aminolevulino-de-hidratase, bem estudadas em mamíferos, devido aos efeitos, respectivamente, de organofosforados e do chumbo, vêm sendo investigadas em peixes e outros organismos. ${ }^{55-57}$

A atividade de algumas enzimas envolvidas no metabolismo intermediário de diversas vias como ATPases e fosfatases tem sido avaliada, ${ }^{39,58-61}$ assim como de enzimas associadas ao estudo do estresse oxidativo como a ascorbato peroxidase, ${ }^{62,63}$ superóxido dismutase,,${ }^{64,65}$ catalase,,${ }^{62,66}$ peroxidase,${ }^{67}$ glutationa sintetase,${ }^{68}$ glutationa redutase e glutationa peroxidase. ${ }^{62,64,69,70}$

\section{MECANISMOS DE INIBIÇÃO E DE AUMENTO DA ATIVIDADE ENZIMÁTICA POR POLUENTES}

A inibição competitiva (Figura 1A) ocorre quando o agente tóxico compete com o substrato na sua interação com enzima. Neste caso, a ação catalítica é reduzida devido à diminuição da proporção de moléculas de enzima que se ligam ao substrato em função do tempo.

Por outro lado, na inibição não-competitiva (Figura 1B), o inibidor e o substrato podem se ligar simultaneamente à enzima. Entretanto, esta ligação do inibidor incapacita a ação catalítica da enzima. Portanto, o inibidor não-competitivo diminui a velocidade máxima de ação enzimática porque diminui a concentração total de enzima ativa. Este último tipo de inibição está relacionado à interação $\mathrm{Hg}$-SH que tem sido observada para a creatina quinase de peixes e para a fosfatase alcalina de crustáceos. ${ }^{71,72}$ Entretanto, uma inibição do tipo competitiva por esse metal foi também observada. ${ }^{71} \mathrm{~A}$ inibição enzimática observada na presença de metais pode ser explicada pela sua interação com grupos - $\mathrm{SH}$ essenciais para a catálise. ${ }^{48}$ É importante salientar que a ligação de tais metais à enzima, em geral, ocorre de maneira irreversível e os dois tipos de inibição, acima mencionados, são exemplos de inibições reversíveis.

Encontram-se amplamente relatados na literatura exemplos de inibições competitivas, não reversíveis, como é o caso de acetilcolinesterases por inseticidas organosfosforados e carbamatos. ${ }^{73,74}$

Vincenzini et al. $^{75}$ relataram as propriedades de detergentes aniônicos de cadeia longa, como o SDS, em inibir competitivamente algumas enzimas, como a lactato desidrogenase de mamíferos e a glicose-6-fosfato desidrogenase de leveduras. De acordo com estes autores, ambos os substratos para estas enzimas, piruvato e glicose6-fosfato, possuem uma ou duas cargas negativas concentradas em pontos específicos da molécula. Talvez pelo fato do detergente possuir uma cabeça hidrofílica carregada negativamente se comportasse como um inibidor competitivo. Este mecanismo poderia explicar a inibição in vitro de várias enzimas pelo detergente aniônico alquilbenzeno linear sulfonado (LAS). ${ }^{76}$

Um outro mecanismo de inibição da atividade poderia ser devido à deficiência de um metal essencial em metaloproteínas ou complexos metálicos de proteínas, onde haveria substituição do metal deficiente pelo metal tóxico ${ }^{60}$ Segundo Joseph et al.,${ }^{77}$ a ligação de um metal ao fosfato orgânico contido no substrato impediria a ação da enzima na liberação do mesmo.

O acréscimo da atividade enzimática, observado pela exposição dos organismos aquáticos frente à ação de alguns poluentes, poderia ocorrer por uma indução da enzima pelo agente tóxico como parte de uma adaptação bioquímica às necessidades metabólicas aumentadas pelo estresse provocado pelo mesmo. ${ }^{30}$ Uma outra suposição, para o caso de metais, é de que o organismo seria capaz de sintetizar proteínas que se ligam a elementos como um mecanismo de detoxificação. ${ }^{60}$

A ativação enzimática poderia ainda estar associada à direta interação do poluente com a enzima, facilitando a interação com o substrato e promovendo alterações das constantes cinéticas, tais como, o a aumento da velocidade máxima ( Vmax) e diminuição da constante de Michaelis (Km) ${ }^{29}$ 


\section{Estudos in vitro}

Estes estudos são realizados pela adição do poluente num sistema de reação contendo o substrato juntamente com a enzima, extraída de uma célula ou tecido supostamente não exposto à ação do contaminante.

Os estudos de avaliação da atividade enzimática in vitro representam uma ferramenta útil na triagem de vários agentes poluentes e têm sido usados em áreas de monitoramento, ${ }^{78,79}$ como métodos de análise semiquantitativa de poluentes orgânicos e metais pesados..$^{80-84}$ Neste sentido, com base no conhecimento da interação poluente-enzima, biossensores para a detecção de metais pesados e agroquímicos foram construídos com fundamentos na inibição da fosfatase alcalina presente em algas. ${ }^{85,86}$ Portanto, tem se demonstrado que a medida da atividade enzimática pode ser usada como um bioindicador da presença de poluentes, consumindo menor tempo de análise e requerendo menores recursos financeiros. ${ }^{31,32}$ Além disto, devemos considerar que a qualidade do efeito para vários agentes químicos tende a ser semelhante à que ocorre no organismo vivo..$^{33,87}$

Dados de testes in vitro podem proporcionar informações úteis para a elucidação do mecanismo de ação tóxica sobre uma determinada espécie de organismo, além de permitirem comparações sobre a susceptibilidade de uma enzima para diferentes poluentes, ${ }^{31,56} \mathrm{ou}$ ainda, sobre a susceptibilidade de diferentes enzimas para um dado poluente. ${ }^{28,88}$ Para isto, determinam-se parâmetros da relação concentração-efeito ou parâmetros cinéticos, tais como, concentração do composto que reduz a atividade enzimática em $50 \%$ ou concentração de inibição média $\left(\mathrm{CI}_{50}\right),{ }^{32,87}$ constante de inibição (Ki) e constante de dissociação $(\mathrm{Kd}) .^{72,89,90} \mathrm{Ou}$ ainda, avaliam-se as alterações na velocidade máxima (Vmax ) e constante de Michaelis (Km). ${ }^{29,79}$

Na Tabela 1 estão apresentados alguns resultados da avaliação de parâmetros de inibição referentes a estudos in vitro com enzimas extraídas de diferentes organismos aquáticos.

Tabela 1. Valores de concentração de inibição média e constante de inibição de poluentes para enzimas de organismos aquáticos

\begin{tabular}{lccccc}
\hline Enzima & Organismo & Poluente & $\mathrm{CI}_{50}(\mathrm{mM})$ & $\mathrm{Ki}(\mathrm{mM})$ & $\mathrm{REF}$ \\
\hline Fosfatase ácida & alga & arsênio & - & 0,023 & 91 \\
& & molibdato & - & 0,6 & 91 \\
Fosfatase alcalina & crustáceo & mercúrio & - & 0,036 & 72 \\
& peixe & vanádio & - & 0,2 & 89 \\
& & mercúrio & 0,53 & - & 87 \\
& & cádmio & 0,24 & - & 87 \\
Adenilciclase & molusco & chumbo & 0,025 & - & 92 \\
Carboxilesterase & peixe & estanho & $>2$ & - & 93 \\
Acetilcolinesterase & peixe & mercúrio & 0,12 & - & 87 \\
& & cádmio & 6,2 & - & 87 \\
& & metil-paraoxon & $123 \times 10^{-6}$ & - & 94 \\
Butirilcolinesterase & peixe & clorpirifós-oxon & $2 \times 10^{-9}$ & - & 95 \\
Glutationa-S- & peixe & mercúrio & 0,5 & - & 87 \\
transferase & & cádmio & 7,1 & - & 87 \\
Anilina hidroxilase & peixe & mercúrio & - & 0,23 & 96 \\
& & níquel & - & 0,43 & 96 \\
& & cádmio & - & 0,65 & 96 \\
Porfobilinogênio & peixe & chumbo & - & 1,3 & 97 \\
sintase & & zinco & - & 1,3 & 97 \\
& & magnésio & - & 3,5 & 97 \\
\hline
\end{tabular}

\section{Estudos in vivo}

São realizados através da medida da atividade da enzima, a qual foi extraída de um organismo-teste submetido ao agente tóxico sob um dado período de tempo. Estes testes têm sido realizados pela exposição de organismos a concentrações conhecidas do poluente, em condições laboratoriais ou de campo, para a avaliação de risco. Portanto, os dados dose-resposta obtidos auxiliam no estabelecimento de níveis aceitáveis de concentração no compartimento aquático. ${ }^{35,39,98}$

A utilização de estudos da alteração da atividade enzimática pela exposição in vivo na avaliação de áreas impactadas e seu monitoramento têm sido extensamente abordadas pela literatura, tanto para poluentes orgânicos como para metais..$^{50,87,99-101}$

A titulo de exemplificação, na Tabela 2 é mostrada a susceptibilidade de várias enzimas após a exposição de algas a poluentes de origem agrícola.

\section{Fosfatases ácidas}

As fosfatases ácidas ou ortofosfato mono éster fosfo-hidrolases (E.C.3.1.3.2.) são enzimas que se caracterizam pela hidrólise de uma grande variedade de ésteres de ortofosfato e reações de transfosforilação, ${ }^{114}$ como esquematizado a seguir:

Hidrólise:

$\mathrm{ROPO}_{3} \mathrm{H}_{2}+\mathrm{H}_{2} \mathrm{O} \rightleftharpoons \mathrm{ROH}+\mathrm{PO}_{4} \mathrm{H}_{3}$

Transfosforilação:

$\mathrm{ROPO}_{3} \mathrm{H}_{2}+\mathrm{R}^{\prime} \mathrm{OH}^{*} \rightleftharpoons \mathrm{ROH}+\mathrm{R}^{\prime} \mathrm{OPO}_{3} \mathrm{H}_{2}$

* aceptor com função álcool

A fosfatase ácida foi inicialmente observada em eritrócitos, ${ }^{115}$ sendo capaz de hidrolisar a ligação éster do fosfato do monofenilfosfato e monoalquilfosfato em pH ótimo entre 5,0 e 6,0, ao contrário da fosfatase alcalina de glóbulos brancos que catalisa essa reação com um pH ótimo de 8,8 a 9,0. Inicialmente a diferença entre as fosfatases ácidas e alcalinas era baseada somente no $\mathrm{pH}$ ótimo para a atividade enzimática. Neuman observou que o mecanismo de reação era diferente para as duas enzimas, ${ }^{116}$ onde a fosfatase ácida requeria para a sua atividade um oxigênio entre o radical e o fosfato. Chaimovich e Nome relataram a atividade das fosfatases ácidas com ausência de cátions mono e bivalentes no meio de reação, enquanto as fosfatases alcalinas requerem metais, principalmente $\mathrm{Mg}^{2+}$, para sua atividade. ${ }^{117}$

\section{Função das fosfatases ácidas e os efeitos de poluentes}

Os lisossomos e as membranas celulares são os primeiros alvos de efeitos de poluentes, uma vez que os lisossomos estão associados com a desintegração de material estranho, e a membrana celular é a primeira barreira encontrada pelo xenobiótico. Portanto, muitos agentes tóxicos e outros fatores estressantes induzem alterações nas membranas lisossomais levando a sua desestabilização. ${ }^{118}$ Esta causa o extravasamento de enzimas hidrolíticas do compartimento lisossomal para o citosol. Portanto, a associação entre hidrolases lisossomais e a membrana lisossomal resulta no fato de que a maior parte da atividade enzimática está normalmente em estado de latência e que se, por alguma razão, a membrana lisossomal se desestabiliza, as enzimas extravasam para o citoplasma com consequente autólise celular. ${ }^{119,120}$

A fosfatase ácida é considerada uma hidrolase lisossomal biomarcadora sendo que, em situações de estresse celular, ocorre extravasamento da enzima para fluidos intra e extracelulares ocasionando a alteração de seus níveis, que pode ser tratada como indicadora de tal estresse. ${ }^{27}$

A transdução de sinal na célula refere-se a qualquer processo em que esta converte um sinal em outro sinal como, por exemplo, o que comanda a síntese de uma determinada proteína. A maioria desses 
Tabela 2. Alteração da atividade de enzimas de algas pela ação de poluentes de origem agrícola

\begin{tabular}{|c|c|c|c|c|}
\hline Enzima & Poluente & Espécie & Efeito & Ref. \\
\hline Ascorbato peroxidase & $\begin{array}{l}\text { Oxifluorfen } \\
\text { Diuron } \\
\mathrm{Cu}^{2+}\end{array}$ & $\begin{array}{c}\text { S. obliquus } \\
\text { S. obliquus } \\
\text { G. tenuistipitata }\end{array}$ & $\begin{array}{c}\text { aumento } \\
\text { inalterado } \\
\text { aumento }\end{array}$ & $\begin{array}{c}62 \\
62 \\
102\end{array}$ \\
\hline ATPase & $\begin{array}{c}\mathrm{Zn}^{2+} \\
\mathrm{Al}^{2+}, \mathrm{Zn}^{2+} \\
\mathrm{Cu}^{2+}\end{array}$ & $\begin{array}{l}\text { S. quadricauda } \\
\text { S. capricornutum } \\
\text { C. vulgaris }\end{array}$ & $\begin{array}{l}\text { diminuição } \\
\text { diminuição } \\
\text { diminuição }\end{array}$ & $\begin{array}{c}60 \\
103 \\
46\end{array}$ \\
\hline eta-D-galactosidase & $\mathrm{Cu}^{2+}, \mathrm{Hg}^{2+}, \mathrm{Cd}^{2+}$ & D. tertiolecta & diminuição & 104 \\
\hline $\mathrm{Ca}^{2+}$ ATPase & $\mathrm{Al}^{3+}$ & C. vulgaris & diminuição & 47 \\
\hline Catalase & $\begin{array}{l}\text { Oxifluorfen } \\
\text { Diuron } \\
\mathrm{Cd}^{2+}, \mathrm{Ni}^{2+}\end{array}$ & $\begin{array}{l}\text { S. obliquus } \\
\text { S. obliquus } \\
\text { S. armatus }\end{array}$ & $\begin{array}{c}\text { aumento } \\
\text { inalterado } \\
\text { aumento }\end{array}$ & $\begin{array}{l}62 \\
62 \\
67\end{array}$ \\
\hline Elongase de sintese de ac. graxos & Thenychlor & Scenedesmus sp & diminuição & 44 \\
\hline Esterase & $\begin{array}{l}\mathrm{Cu}^{2+} \\
\mathrm{LAS}\end{array}$ & $\begin{array}{l}\text { S. capricornutum } \\
\text { N. gaditana }\end{array}$ & $\begin{array}{l}\text { diminuição } \\
\text { diminuição }\end{array}$ & $\begin{array}{l}105 \\
106\end{array}$ \\
\hline Ferridoxina nitrato redutase & $\mathrm{Cd}^{2+}$ & C. reinhardtii & inalterado & 107 \\
\hline Fitoquelatina sintase & $\mathrm{Zn}^{2+}, \mathrm{Cd}^{2+}$ & D. tertiolecta & aumento & 68 \\
\hline Fosfatase alcalina & $\begin{array}{l}\text { Dimetoato } \\
\mathrm{Hg}^{2+}, \mathrm{Pb}^{2+}\end{array}$ & $\begin{array}{l}\text { C. vulgaris } \\
\text { S. bijuga }\end{array}$ & $\begin{array}{c}\text { aumento } \\
\text { diminuição }\end{array}$ & $\begin{array}{c}108 \\
39\end{array}$ \\
\hline Fosfatase ácida & $\begin{array}{l}\text { Hexaclorobenzeno } \\
\qquad \mathrm{Hg}^{2+}, \mathrm{Pb}^{2+}\end{array}$ & $\begin{array}{c}\text { S. capricornutum } \\
\text { S. bijuga }\end{array}$ & $\begin{array}{l}\text { diminuição } \\
\text { diminuição }\end{array}$ & $\begin{array}{c}109 \\
39\end{array}$ \\
\hline Gama-glutamil-cisteina sintetase & $\mathrm{Zn}^{2+}, \mathrm{Cd}^{2+}$ & D. tertiolecta & aumento & 68 \\
\hline Glicose-6-fosfato-desidrogenase & $\begin{array}{l}\text { Hexaclorobenzeno } \\
\qquad \mathrm{Al}^{3+}\end{array}$ & $\begin{array}{l}\text { S. capricornutum } \\
\text { S. capricornutum }\end{array}$ & $\begin{array}{l}\text { diminuição } \\
\text { diminuição }\end{array}$ & $\begin{array}{l}109 \\
103\end{array}$ \\
\hline Glutamato desidrogenase & $\mathrm{Hg}^{2+}, \mathrm{Pb}^{2+}$ & S. bijuga & aumento & 39 \\
\hline Glutamina sintetase & $\mathrm{Cd}^{2+}$ & C. reinhardtii & diminuição & 107 \\
\hline Glutationa peroxidase & $\mathrm{Ni}^{2+}$ & S. acutus & aumento & 64 \\
\hline Glutationa redutase & $\begin{array}{l}\text { Oxifluorfen } \\
\text { Diuron } \\
\mathrm{Cd}^{2+}\end{array}$ & $\begin{array}{l}\text { S. obliquus } \\
\text { S. obliquus } \\
\text { N. oculata }\end{array}$ & $\begin{array}{c}\text { aumento } \\
\text { inalterado } \\
\text { diminuição }\end{array}$ & $\begin{array}{c}62 \\
62 \\
110\end{array}$ \\
\hline Glutationa sintetase & $\mathrm{Zn}^{2+}, \mathrm{Cd}^{2+}$ & D. tertiolecta & aumento & 68 \\
\hline Glutationa-S-transferase & $\begin{array}{l}\text { Oxifluorfen } \\
\text { Diuron }\end{array}$ & $\begin{array}{l}\text { S. obliquus } \\
\text { S. obliquus }\end{array}$ & $\begin{array}{l}\text { aumento } \\
\text { inalterado }\end{array}$ & $\begin{array}{l}62 \\
62\end{array}$ \\
\hline Malato desidrogenase & $\mathrm{Hg}^{2+}, \mathrm{Pb}^{2+}$ & S. bijuga & diminuição & 39 \\
\hline $\mathrm{Mg}^{++}$ATPase & $\mathrm{A}^{3+}$ & C. vulgaris & diminuição & 47 \\
\hline $\mathrm{NAD}^{+}$isocitrato desidrogenase & $\mathrm{Cd}^{2+}$ & C. reinhardtii & aumento & 111 \\
\hline NADH glutamato sintetase & $\mathrm{Cd}^{2+}$ & C. reinhardtii & inalterado & 107 \\
\hline $\mathrm{NADP}^{+}$isocitrato desidrogenase & $\mathrm{Cd}^{2+}$ & C. reinhardtii & aumento & 111 \\
\hline NADPH óxido redutase & $\mathrm{Hg}^{2+}, \mathrm{Cd}^{2+}, \mathrm{Zn}^{2+}$ & E. gracilis & diminuição & 48 \\
\hline Nitrato redutase & $\begin{array}{l}\mathrm{Al}^{3+} \\
\mathrm{Zn}^{2+}\end{array}$ & $\begin{array}{c}\text { C. vulgaris } \\
\text { S. capricornutum }\end{array}$ & $\begin{array}{l}\text { diminuição } \\
\text { diminuição }\end{array}$ & $\begin{array}{c}47 \\
103\end{array}$ \\
\hline O-acetil-L-serina(tiol)liase & $\begin{array}{l}\mathrm{Cd}^{2+} \\
\mathrm{Cd}^{2+}\end{array}$ & $\begin{array}{l}\text { C. reinhardtii } \\
\text { C. reinhardtii }\end{array}$ & $\begin{array}{l}\text { aumento } \\
\text { inalterado }\end{array}$ & $\begin{array}{l}111 \\
107\end{array}$ \\
\hline Peroxidase & $\mathrm{Cd}^{2+}, \mathrm{Ni}^{2+}, \mathrm{Mn}^{2+}$ & S. armatus & aumento & 67 \\
\hline Protoclorofilida redutase & $\mathrm{Hg}^{2+}, \mathrm{Cd}^{2+}$ & E. gracilis & diminuição & 48 \\
\hline Protoporfirinogênio IX oxidase & Tiosemicarbazidas & S. acutus & diminuição & 112 \\
\hline Serina acetil transferase & $\mathrm{Cd}^{2+}$ & C. reinhardtii & aumento & 111 \\
\hline Superóxido dismutase & $\begin{array}{c}\mathrm{Cu}^{2+} \\
\mathrm{Ni}^{2+} \\
\text { Antraceno } \\
\mathrm{Cd}^{2+}\end{array}$ & $\begin{array}{l}\text { G. tenuistipitata } \\
\text { S. acutus } \\
\text { S. subspicatus } \\
\text { S. subspicatus }\end{array}$ & $\begin{array}{l}\text { aumento } \\
\text { diminuição } \\
\text { aumento } \\
\text { aumento }\end{array}$ & $\begin{array}{c}102 \\
64 \\
113 \\
113\end{array}$ \\
\hline Transaminase glutâmico oxaloacética & $\mathrm{Pb}^{2+}$ & S. bijuga & diminuição & 39 \\
\hline Transaminase glutâmico pirúvica & $\mathrm{Hg}^{2+}, \mathrm{Pb}^{2+}$ & S. bijuga & diminuição & 39 \\
\hline Urease & $\mathrm{Cu}^{2+}$ & C. vulgaris & diminuição & 46 \\
\hline
\end{tabular}

eventos envolve uma sequência ordenada de reações executada por enzimas, que resulta numa via de transdução de sinal. Assim sendo, as fosfatases ácidas podem atuar na desfosforilação de receptores de moléculas sinalizadoras, como a insulina, na regulação do processo de transdução de sinal. ${ }^{121}$ Neste contexto, as fosfatases ácidas participam em processos de sinalização celular como, por exemplo, os que envolvem o ácido ascórbico na resposta a condições fotoxidativas e ataques de patógenos em plantas. ${ }^{122}$

Na Tabela 3 estão apresentados alguns dados da literatura referentes à alteração da atividade desta enzima em diversos organismos aquáticos. Entre estes, os peixes têm sido alvo de estudo em muitos trabalhos que tratam de efeitos de poluentes na fosfatase ácida (Tabela 4), 
Tabela 3. Efeito de poluentes de origem agrícola na fosfatase ácida de diversos organismos aquáticos

\begin{tabular}{|c|c|c|c|c|c|}
\hline Organismo & Poluente & Sistema de exposição & Origem & Efeito na atividade & Ref. \\
\hline \multicolumn{6}{|l|}{ FUNGO } \\
\hline A. proliferiodes & clortalonil & in vivo & organismo todo & aumento & 123 \\
\hline D. sterilis & clortalonil & in vivo & organismo todo & diminuição & 123 \\
\hline \multicolumn{6}{|l|}{ PROTOZOÁRIO } \\
\hline T. pyriformis & cobre & in vivo & organismo todo & aumento/diminuição & 124 \\
\hline PLANTA & cobre & & & & \\
\hline S. oligorrhiza & & in vitro & organismo todo & inalterado & 125 \\
\hline \multicolumn{6}{|l|}{ CRUSTACEO } \\
\hline D. magna & cobre & in vivo & organismo todo & diminuição & 126 \\
\hline Camarão & aldrin, lindano & in vitro & vários tecidos & diminuiçãão & 127 \\
\hline Artemia & metilparation & in vivo & hepatopâncreas & aumento & 127 \\
\hline \multirow[t]{5}{*}{ Caranguejo } & diclorvos & in vivo & vários tecidos & aumento & 128 \\
\hline & endosulfan & in vivo & varios tecidos & aumento/diminuição & 129 \\
\hline & cipermetrina, endosulfan & in vitro & organismo todo & diminuição & 31 \\
\hline & metomyl, clorpirifós & in vitro & organismo todo & diminuiç̧ão & 31 \\
\hline & monocrotofós & in vitro & organismo todo & diminuição & 130 \\
\hline \multicolumn{6}{|l|}{ MOLUSCO } \\
\hline \multirow[t]{3}{*}{ Bivalve } & metais em estuário & in vivo & digestivo e rim & diminuição & 131 \\
\hline & zinco, chumbo & in vivo & hemócitos & diminuição & 132 \\
\hline & cobre & in vivo & $\begin{array}{c}\text { brânquias/glândula digestiva } \\
\text { organismo todo }\end{array}$ & aumento & 133 \\
\hline \multirow[t]{3}{*}{ Gastrópodo } & aldicarb, aldoxycarb & in vivo & organismo todo & diminuição & 134 \\
\hline & metomyl, oxamyl & in vivo & hemolinfa & diminuição & 134 \\
\hline & cobre & in vivo & & aumento & 27 \\
\hline \multicolumn{6}{|l|}{ INSETO } \\
\hline Hemiptera & cobre, chumbo & in vivo & organismo todo & aumento & 135 \\
\hline
\end{tabular}

Tabela 4. Efeito de poluentes de origem agrícola sobre a fosfatase ácida de peixes

\begin{tabular}{|c|c|c|c|c|c|}
\hline Poluente & Sistema de exposição & Tecido & Espécie & Efeito na atividade & Ref \\
\hline Mistura de pesticidas, metais, $\mathrm{PCBs} *$, $\mathrm{PAHs} * *$ & in vitro & hepatócitos isolados & O. mykiss & aumento & 66 \\
\hline Mistura de pesticidas, PCBs, PAHs & in vitro & hepatócitos isolados & O. mykiss & diminuição & 136 \\
\hline Deltametrina & in vivo & fígado & O.mossambicus & diminuição & 137 \\
\hline Aldicarb & $\begin{array}{l}\text { in vivo } \\
\text { in vitro }\end{array}$ & $\begin{array}{l}\text { intestino } \\
\text { intestino }\end{array}$ & $\begin{array}{l}\text { P. conchonius } \\
\text { P. conchonius }\end{array}$ & $\begin{array}{l}\text { inalterado } \\
\text { inalterado }\end{array}$ & 33 \\
\hline Endosulfan & $\begin{array}{l}\text { in vivo } \\
\text { in vivo } \\
\text { in vitro }\end{array}$ & $\begin{array}{c}\text { fígado, músculo } \\
\text { ovário } \\
\text { ovário }\end{array}$ & $\begin{array}{c}\text { C. gachua } \\
\text { P. conchonius } \\
\text { P. conchonius }\end{array}$ & $\begin{array}{l}\text { diminuição } \\
\text { aumento } \\
\text { inalterado }\end{array}$ & $\begin{array}{r}138 \\
33 \\
33\end{array}$ \\
\hline Quinalfós & in vivo & cérebro & L. rohita & aumento & 139 \\
\hline Cipermetrina & in vivo & fígado & H. fossilis & diminuição & 59 \\
\hline Dimetoato & in vivo & $\begin{array}{l}\text { fígado } \\
\text { músculo }\end{array}$ & $\begin{array}{l}\text { L. thermalis } \\
\text { L. thermalis }\end{array}$ & $\begin{array}{l}\text { aumenta } \\
\text { aumenta }\end{array}$ & $\begin{array}{l}140 \\
140\end{array}$ \\
\hline Malation & in vivo & fígado & B. rerio & diminuição & 141 \\
\hline Monocrotofós & in vivo & plasma & C. punctatus & aumento & 142 \\
\hline p-t-butilfenol & in vivo & músculo & C. carpio & aumento & 143 \\
\hline Toxafeno & in vivo & hepatócitos isolados & P. ferrugineus & aumento & 144 \\
\hline Disulfoton + endosulfan & in vivo & fígado & O. mykiss & diminuição & 38 \\
\hline LAS & $\begin{array}{l}\text { in vivo } \\
\text { in vivo }\end{array}$ & $\begin{array}{l}\text { brânquias, fígado } \\
\text { intestino, rim }\end{array}$ & $\begin{array}{l}\text { C. carpius } \\
\text { M. vittatus }\end{array}$ & $\begin{array}{l}\text { diminuição } \\
\text { diminuição }\end{array}$ & $\begin{array}{l}145 \\
146\end{array}$ \\
\hline Cobre & $\begin{array}{l}\text { in vivo } \\
\text { in vitro } \\
\text { in vitro } \\
\text { in vivo }\end{array}$ & $\begin{array}{l}\text { intestino } \\
\text { intestino } \\
\text { fígado } \\
\text { fígado }\end{array}$ & $\begin{array}{l}\text { P. conchonius } \\
\text { P. conchonius } \\
\text { P. conchonius } \\
\text { P. conchonius }\end{array}$ & $\begin{array}{c}\text { aumento } \\
\text { inalterado } \\
\text { inalterado } \\
\text { diminuição }\end{array}$ & $\begin{array}{l}28 \\
28 \\
28 \\
28\end{array}$ \\
\hline Cádmio & $\begin{array}{l}\text { in vivo } \\
\text { in vitro } \\
\text { in vivo }\end{array}$ & $\begin{array}{c}\text { ovário } \\
\text { ovário } \\
\text { soro }\end{array}$ & $\begin{array}{l}\text { B. conchonius } \\
\text { B. conchonius } \\
\text { L. macrochirus }\end{array}$ & $\begin{array}{c}\text { aumento } \\
\text { inalterado } \\
\text { aumento }\end{array}$ & $\begin{array}{r}30 \\
30 \\
147\end{array}$ \\
\hline Mercúrio & in vivo & fígado, brânquias & N. notopterus & diminuição & 148 \\
\hline
\end{tabular}

*Bifenilas policloradas. **Hidrocarbonetos aromáticos policíclicos 
talvez pelo fato de serem os organismos mais representativos e de maior importância econômica nos ecossistemas aquáticos.

As Tabelas 3 e 4 demonstram que os efeitos são de natureza diversa podendo ocorrer aumento, diminuição ou mesmo, não alteração da atividade fosfatásica. Estas respostas são particulares para cada agente químico em relação ao sistema de exposição (in vitro ou in vivo), tecido e organismo. Isto estaria relacionado a que cada espécie é caracterizada por um padrão específico de isoenzimas, tal como a variabilidade em isoformas de fosfatase ácida que ocorre entre oito espécies de fungos aquáticos do gênero Achlya. ${ }^{149}$

Pelo motivo de serem as algas componentes da base da cadeia alimentar nos ecossistemas aquáticos, aliado ao envolvimento das fosfatases no metabolismo de um elemento, o fósforo, de extrema importância para a viabilidade desses organismos, serão abordados alguns aspectos sobre as fosfatases ácidas de algas e sua resposta à ação de agentes tóxicos.

\section{Fosfatases ácidas de algas e sua susceptibilidade a poluentes}

Várias funções têm sido atribuídas a estas enzimas em algas, tais como a participação em processos autofágicos digestivos e hidrólise de material fosfolipídico; ${ }^{150}$ rompimento de plasmalema e absorção do flagelo durante a fertilização; reciclagem de fosfato inorgânico para sua reassimilação $;{ }^{151-153}$ diferenciação dos esporos; ${ }^{154}$ transporte de substâncias através de membranas e disponibilidade de fosfato inorgânico a partir do meio extracelular. ${ }^{155,156}$ Esta última função também é desempenhada pela fosfatase alcalina, cuja atividade é aumentada pela exposição das algas em um meio de cultura deficiente em fosfato inorgânico. ${ }^{157-159}$

Os trabalhos sobre o aumento de síntese de fosfatases ácidas em algas pela ausência de fosfato inorgânico no meio de cultura, assim como o estudo das propriedades destas fosfatases induzidas e das constitutivas, datam da década de 1960 em pesquisas realizadas com Euglena gracilis e Chlorella pyrenoidosa. ${ }^{91,160}$

A extração da fosfatase ácida de algas do gênero Selenastrum foi relatada por Kong e Chen e por Kong et al. utilizando tampão Trisborato $0,1-0,3 \mathrm{M} \mathrm{pH} 7,5$ e centrifugação a 10.000 x $g \cdot{ }^{103,109}$ Entretanto, os autores não descreveram o método de ruptura celular utilizado. Para este procedimento, técnicas que utilizam ultrassom foram descritas na extração de fosfatases das algas cloroficeas Chlamydomonas e Chlorella vulgaris. ${ }^{47,108,161,162}$ Patni e Aaronson usaram pérolas de vidro e centrifugação para extrair a enzima de Ochromonas danica ${ }^{163}$ Esta enzima também pode ser extraída em uma preparação de pó cetônico quando um precipitado algáceo é tratado com acetona à temperatura de -20 a $-30{ }^{\circ} \mathrm{C} .{ }^{91,160,164}$

A alga verde unicelular Chlamydomonas reinhardtii tem sido adotada em vários trabalhos como organismo modelo para o estudo de fosfatases. Esta alga produz duas formas principais de fosfatases ácidas constitutivas que diferem quanto a sua sensibilidade à inativação térmica, ${ }^{161}$ as quais estão invariavelmente presentes em células cultivadas na ausência ou presença de fosfato inorgânico com atividade máxima em pH de aproximadamente 5,0.159,162 Quando o extrato dessa mesma espécie, crescida na presença de $\mathrm{Pi}$, é submetido à eletroforese de isoeletrofocalização, ocorre o aparecimento de três bandas referentes a fosfatases ácidas com diferentes valores de pontos isoelétricos. ${ }^{165}$

As fosfatases ácidas de algas também têm sido caracterizadas quanto a sua especificidade para diferentes substratos, efeito de metais na atividade, ${ }^{163,166}$ valor de Km para um dado substrato e energia de ativação. ${ }^{91,163,164}$ Entretanto, existe uma carência de informações na literatura quanto às propriedades destas enzimas e sua utilização como bioindicadoras de efeitos de poluentes.

Em algas, as fosfatases ácidas estão localizadas em vários compartimentos celulares. Segundo Cooper et al., ${ }^{150}$ a enzima está presente em vacúolos, dispersa no citoplasma e em pontos de degradação focal. A enzima também foi encontrada no complexo de Golgi, ${ }_{150,154}$ mitocôndria, ${ }^{155}$ membrana de tilacoides, ${ }^{155}$ sistema de endomembrana, ${ }^{152}$ membrana externa e corpos-PAS. ${ }^{155,156,167}$ Segundo Tornqvist, ${ }^{155}$ a localização da atividade fosfatásica ácida é alterada do citoplasma para a parede celular quando algas cloroficeas são expostas a íons metálicos, como $\mathrm{Al}^{3+}$.

Patni e Aaronson, ${ }^{163}$ estudando os efeitos in vitro em sistema pré-incubado, observaram que a fosfatase ácida intracelular extraída de Ochromonas danica é mais susceptível à inibição pelo $\mathrm{Hg}^{2+}$, enquanto que a extracelular é mais sensível à inibição pelo $\mathrm{Cu}^{2+}$. Em um sistema de exposição semelhante, a alteração da atividade da enzima extraída de Pseudokirchneriella subcapitata foi avaliada frente a ação de 30 poluentes de origem agrícola, constatando-se que somente o íon $\mathrm{Cu}^{2+}$ possuía efeito ativador. ${ }^{61}$ Este elemento também aumentou a atividade da enzima da macroalga marinha Ulva fasciata quando exposta às concentrações de 50 e $100 \mu \mathrm{M} .{ }^{168}$ Segundo Joseph et al.,${ }^{77}$ um possível mecanismo pelo qual ocorreria o aumento da atividade da enzima em Chlamydomonas reinhardtii exposta ao $\mathrm{Al}^{3+}$ seria explicado pela ligação deste ao fosfato orgânico. Nesta situação, a utilização de fosfato estaria comprometida e a célula induziria a síntese da fosfatase como uma resposta compensatória.

Como foi anteriormente descrito, apesar de alguns estudos relatarem um efeito ativador ou indutor da síntese da enzima na presença do poluente, resultando numa maior atividade, a maioria dos trabalhos aborda os efeitos inibitórios. Kong et al. ${ }^{109}$ observaram uma correlação positiva entre o número de átomos de halogênio em halogenobenzenos e o decréscimo da atividade específica da fosfatase ácida em culturas de Selenastrum capricornutum contendo estes compostos.

Metais pesados considerados altamente tóxicos para algas, tais como $\mathrm{Hg}^{2+} \mathrm{e} \mathrm{Cu}^{2+}$, demonstraram a capacidade de diminuir a atividade da fosfatase ácida destes organismos quando expostos a pequenas concentrações. Assim, por exemplo, aproximadamente $90 \%$ desse parâmetro foi reduzido por $10 \mu \mathrm{M}$ de $\mathrm{Hg}^{2+}$ em Scenedesmus bijuga. ${ }^{39} \mathrm{O} \mathrm{Cu}^{2+}$, por sua vez, diminui a atividade especifica em $C$. vulgaris em aproximadamente $50 \%$ quando a alga foi exposta a 31 $\mu \mathrm{M} .{ }^{169}$ Porém, isto foi observado em culturas crescidas em $\mathrm{pH} 4,0$, sendo que somente cerca de $20 \%$ da atividade foi afetada em pH 6,8.

Elementos metálicos com maiores concentrações permissíveis na água e maiores valores de concentração efetiva média $\left(\mathrm{CE}_{50}\right.$ ou concentração que inibe o crescimento algáceo em 50\%) para algas também demonstraram alterar a atividade quando testados na concentração da ordem de $100 \mu \mathrm{M}$ ou bem inferior a esta. Assim, o efeito na atividade específica pelo $\mathrm{Zn}^{2+}$ em estudos com Scenedesmus obliquus e Selenastrum capricornutum demonstraram que a diminuição de aproximadamente $50 \%$ desse parâmetro ocorria nas concentrações de $120 \mu \mathrm{M}$ (10 dias de exposição) e $4 \mu \mathrm{M}$ (4 dias de exposição), respectivamente. ${ }^{60,103}$ Porém, observou-se um aumento da atividade específica superior a $200 \%$ em Scenedesmus quadricauda exposta a $70 \mu \mathrm{M}$ desse metal. ${ }^{60}$

Uma redução significativa, de aproximadamente $20 \%$, da atividade da enzima foi constatada em culturas de Chlorella vulgaris expostas a $150 \mu \mathrm{M}$ de $\mathrm{Al}^{3+}$ durante 15 dias. ${ }^{47} \mathrm{~A}$ mesma porcentagem de redução foi obtida com uma concentração 1000 vezes menor desse metal em $S$. capricornutum exposta por 4 dias. ${ }^{103} \mathrm{O} \mathrm{Ni}^{2+}$, por sua vez, diminuiu a atividade específica em cerca de $20 \%$ em C. vulgaris exposta a $34 \mu \mathrm{M},{ }^{169}$ enquanto que o $\mathrm{Pb}^{2+}$ reduziu a atividade na ordem de $60 \%$ em S. bijuga exposta a $10 \mu \mathrm{M}^{39}$

$\mathrm{O}$ efeito inibidor interativo entre $\mathrm{Al}^{3+} \mathrm{e} \mathrm{F}^{-}$sobre a fosfatase de $C$. vulgaris, cultivada na presença de ambos metais, demonstrou ser sinérgico para a forma ácida, enquanto que foi aditivo para a forma alcalina. ${ }^{47}$

A alteração de fatores físico-químicos no meio de cultura, como a aplicação de radiação UV-B e diminuição do $\mathrm{pH},{ }^{46,169}$ aumentou o efeito inibitório do $\mathrm{Cu}^{2+}$ sobre a atividade específica da fosfatase ácida. 


\section{CONSIDERAÇÕES FINAIS}

No Brasil, a legislação que regula a emissão e a concentração máxima permissível de agroquímicos, assim como de outros compostos tóxicos, em corpos de águas superficiais para a preservação das comunidades aquáticas está fundamentada na Resolução № 357 de 17/03/05 do CONAMA - Conselho Nacional do Meio Ambiente. ${ }^{170}$ Entretanto, esta resolução especifica concentrações máximas permissíveis para diferentes classes de água somente para aproximadamente menos de 30 compostos de uso agrícola, sendo que alguns destes produtos já foram retirados do mercado e, aparentemente, estão em desuso. Os parâmetros de níveis aceitáveis nessa legislação, tanto para agrotóxicos como para outros poluentes, têm sido propostos com base em legislações internacionais. Além disso, devemos considerar que a maioria desses parâmetros têm sido proposta com base em efeitos não tão sutis, como são as alterações em componentes bioquímicos. Deste modo, a avaliação da atividade enzimática dos diferentes níveis tróficos representa um instrumento importante na avaliação de risco em nível laboratorial ou em campo.

O uso de parâmetros biológicos para medir a qualidade da água de um sistema supostamente impactado como, por exemplo, parâmetros de natureza bioquímica, baseia-se nas respostas dos organismos em relação ao meio onde vivem. Neste sentido, sendo o Brasil um país eminentemente agrícola constata-se o apreciável risco de áreas sujeitas à degradação, incluindo os compartimentos aquáticos. Assim sendo, a utilização de avaliações de atividade enzimática em organismos supostamente expostos à ação de xenobióticos constitui uma ferramenta relevante para fins de monitoramento.

\section{REFERÊNCIAS}

1. Murty, A. S. Em Toxicity of Pesticides to Fish; Murty, A. S., ed.; CRC Press: Boca Raton, 1986, vol. 1.

2. Bedor, C. N. G.; Ramos, L. O.; Rego, M. A. V.; Pavão, A. C.; Augusto, L. G. S.; Rev. Baiana Saúde Públ. 2007, 31, 68.

3. Garcia, E. G.; Bussacos, M. A.; Fischer, F. M.; Rev. Saúde Públ. 2005, $39,832$.

4. Verma, S. R.; Bansal, S. K.; Gupta, A. K.; Pal, N.; Tyagi, A. K.; Bhatnagar, M. C.; Kumar, V.; Dalela, R. C.; Water Res. 1982, 16, 525.

5. Jonsson, C. M.; Toledo, C. M. F.; Arch. Environ. Contam. Toxicol. 1993, $24,151$.

6. Rocha, M. T.; Dissertação de Mestrado, Universidade de São Paulo, Brasil, 1998.

7. Singh, R. P.; Agrawal, M.; Waste Manag. 2008, 28, 347.

8. Mattiazzo, M. E.; Berton, R. S.; Cruz, M. C. P. Em Micronutrientes e Elementos Tóxicos na Agricultura; Ferreira, M. E.; Cruz, M. C. P.; van Raij, B.; Abreu, C. A., eds.; CNPq/FAPESP/POTAFOS: Jaboticabal, 2001.

9. Erhardt, W.; Prüeß, A.; Organic Contaminants in Sewage Sludge for Agriculture Use, European Commision, Joint Research Center, UMEG Center for Environmental Measurements: Karlsruhe, 2001.

10. Nimmo, D. R. Em Fundamentals of Aquatic Toxicology: Methods and Application; Rand, G. M.; Petrocelli, S. R., eds.; Hemisphere Publishing Corporation: Washington, 1985.

11. Jergentz, S.; Mugni, H.; Bonetto, C.; Schulz, R.; Arch. Environ. Contam. Toxicol. 2004, 46, 345.

12. Brun, G. L.; Mac Donald, R. M.; Verge, J.; Aubé, J.; Chemosphere 2008, 71, 314.

13. Leland, H. V.; Kuwabara, J. S. Em Fundamentals of Aquatic Toxicology: Methods and Application; Rand, G. M.; Petrocelli, S. R., eds.; Hemisphere Publishing Corporation: Washington, 1985.

14. Singh, S. P.; Tack, F. M. G.; Gabriels, D.; Verloo, M. G.; Water, Air, Soil Pollut. 2000, 118, 73.
15. Pang, X. P.; Letey, J.; Soil Sci. 1999, 164, 922.

16. Ortiz, J. B.; Gonzalez De Canales, M. L.; Sarasquete, C.; Scientia Mar. 2003, 67, 53.

17. Taylor, G. E. Jr.; Schaller, K. B.; Geddes, J. D.; Gustin, M. S.; Environ. Toxicol. Chem. 1996, 15, 1694.

18. Hurtig, A. K.; San Sebastian, M.; Soto, A.; Shingre, A.; Zambrano, D.; Guerrero, W.; Arch. Environ. Health 2003, 58, 223.

19. Felsot, A. S.; Racke, K. D.; Hamilton, D. J.; Rev. Environ. Contam. Toxicol. 2003, 177, 123.

20. Jonsson, C. M.; Maia, A. H. N.; Pesticidas (UFPR) 2007, 17, 1.

21. Jonsson, C. M.; Paraiba, L. C.; Mendoza, M. T.; Sabater, C.; Carrasco, J. M.; Chemosphere 2001, 43, 321.

22. Valavanidis, A.; Vlahogianni, T; Dassenakis, M; Scoullos, M.; Ecotoxicol. Environ. Saf. 2006, 64, 178.

23. Henderson, R. F.; Toxicol. Lett. 1995, 82, 379.

24. Jimenez, B. D.; Stegeman, J. J.; Am. Fish. Soc. Symp. 1990, 8, 67.

25. Payne, J. F.; Fancey, L.; Rahimtula, A.; Porter, E.; Comp. Biochem. Physiol. C. 1987, 86, 233.

26. Netrawali, M. S.; Gandhi, S. R.; Bull. Environ. Contam. Toxicol. 1990, 44, 819.

27. Suresh, P. G.; Reju, M. K.; Mohandas, A.; Sci. Total Environ. 1993, Suppl. 2, 1265.

28. Gill, T. S., Tewari, H.; Pande, J.; Ecotoxicol. Environ. Saf. 1992, 23, 294.

29. Bounias, M.; Kruk, I.; Nectoux, M.; Popeskovic, D.; Ecotoxicol. Environ. Saf. 1996, 35, 67.

30. Gill, T. S.; Tewari, H.; Pande, J.; Comp. Biochem. Physiol. C 1991, 100, 501.

31. Elsebae, A. A.; Alex. Sci. Exch. 1996, 17, 417.

32. El Demerdash, F. M.; Yousef, M. I.; Elagamy, E. I.; J. Environ. Sci. Health B 2001, 36, 29.

33. Gill, T. S.; Pande, J.; Tewari, H.; Pest. Biochem. Physiol. 1990, 38, 231.

34. Tripathi, G.; Verma, P.; Pest. Biochem. Physiol. 2006, 86, 167.

35. Jonsson, C. M.; Ferracini, V. L.; Paraiba, L. C.; Rangel, M.; Aguiar, S. R.; Scientia Agri. 2002, 59, 441.

36. Samuel, M.; Sastry, K. V.; Pest. Biochem. Phisiol. 1989, 34, 1.

37. Surendranath, P.; Ghouselazam, S.; Rao, K. V. R.; Bull. Environ. Contam. Toxicol. 1991, 46, 738.

38. Arnold, H.; Pluta, H. J.; Braunbeck, T.; Aquat. Toxicol. 1995, 33, 17.

39. Fathi, A. A.; Folia Microbiol. 2002, 47, 667.

40. Simon, L. M.; Laszlo, K.; Kotorman, M.; Vertesi, A.; Bagi, K.; Nemcsok, J.; J. Environ. Sci. Health B Pest. Food Contam. Agr. Wastes 1999, 34, 819.

41. Kotorman, M.; Laszlo, K.; Nemcsok, J.; Simon, L. M.; J. Environ. Sci. Health A Toxic Hazar. Subst. Environ. Engin. 2000, 35, 1517.

42. Tugiyono; Gagnon, M. M.; Comp. Biochem. Physiol. C Toxicol. Pharmacol. 2002, 132, 425.

43. Singh, A.; Agarwal, R. A.; Bull. Environ. Contam. Toxicol. 1993, 51, 445.

44. Takahashi, H.; Ohki, A.; Kato, S.; Tanaka, A.; Sato, Y.; Matthes, B.; Boeger, P.; Wakabayashi, K.; Pest. Biochem. Physiol. 2001, 71, 140.

45. Rao, K. V. R.; Surendranath, P.; Pest. Biochem. Physiol. 1991, 39, 205.

46. Rai, P. K.; Rai, L. C.; J. Gen. Appl. Microbiol. 1997, 43, 281.

47. Rai, L. C.; Husaini, Y.; Mallick, N.; Aquat. Toxicol. 1998, $42,67$.

48. van Assche, F.; Clijsters, H.; Plant Cell Environ. 1990, 13, 195.

49. Trebst, A.; Depka, B.; Hollander-Czytko, H.; FEBS Lett. 2002, 516, 156.

50. Fenet, H.; Casellas, C.; Bontoux, J.; Ecotoxicol. Environ. Saf. 1998, 40, 137.

51. Ruus, A.; Sandvik, M; Ugland, K. I.; Skaare J. U.; Aquat. Toxicol. 2002, 61,73 .

52. Ramljak, S.; Hackenberger, B. K.; Smital, T.; Britvic, S.; J. Environ. Sci. Health B, Pest. Food Contam. Agric. Wastes 2000, 35, 751.

53. Taysse, L.; Chambras, C.; Marionnet, D.; Bosgiraud, C.; Deschauux, P.; Bull. Environ. Contam. Toxicol. 1998, 60, 300. 
54. Tarja, N.; Kirsti, E.; Marja L.; Kari E.; Environ. Toxicol. 2003, 18, 219

55. Burden, V. M.; Sandheinrich, M. B.; Caldwell, C. A.; Environ. Pollut 1998, 101, 285.

56. Jin-Clark, Y.; Lydy, M. J.; Zhu, K. Y.; Environ. Toxicol. Chem. 2002, 21, 598.

57. Denton, D. L.; Wheelock, C. E.; Murray, S. A.; Hammock, B. D.; Hinton, D. E.; Environ. Toxicol. Chem. 2003, 22, 336.

58. Glover, C. N.; Petri, D.; Tollefsen, K. E.; Jørum, N.; Handy, R. D.; Berntssen, M. H.; Aquat. Toxicol. 2007, 84, 346.

59. Saha, S.; Kaviraj, A.; Chemosphere 2009, 74, 1254.

60. Omar, H. H.; Int. Biodeter. Biodegrad. 2002, 50, 95.

61. Jonsson, C. M.; Aoyama, H.; Chemosphere 2007, 69, 849.

62. Geoffroy, L.; Teisseire, H.; Couderchet, M.; Vernet, G.; Pest. Biochem. Physiol. 2002, 72, 178.

63. Mallick, N.; J. Plant Physiol. 2004, 161, 591.

64. Randhawa, V. K.; Zhou, F.; Jin, X.; Nalewajko, C.; Kushner, D. J.; Can. J. Microbiol. 2001, 47, 987.

65. Zikic, R. V.; Stajn, A. S.; Pavlovic, S. Z.; Ognjanovic, B. I.; Saicic, Z. S.; Physiol. Res. 2001, 50, 105.

66. Strmac, M.; Braunbeck, T.; Ecotoxicol. Environ. Saf. 2002, 53, 293.

67. El Enany, A. E.; Issa, A. A.; Folia Microbiol. 2001, 46, 227.

68. Tsuji, N.; Hirayanagi, N.; Iwabe, O.; Namba, T.; Tagawa, M.; Miyamoto, S.; Miyasaka, H.; Takagi, M.; Hirata, K.; Miyamoto, K.; Phytochemistry 2003, 62, 453.

69. Pena-Llopis, S.; Pena, J. B.; Sancho, E.; Fernandez-Vega, C.; Ferrando, M. D.; Chemosphere 2001, 45, 671.

70. Elia, A. C.;Galarini, R.; Taticchi, M. I.; Dorr, A. J.; Mantilacc, L.; Ecotoxicol. Environ. Saf. 2003, 55, 162.

71. Araujo, G. M.; Silva, C. B.; Hasson-Voloch, A.; Int. J. Biochem. Cell Biol. 1996, 28, 491.

72. Chen, Q. X.; Zheng, W. Z.; Lin, J. Y.; Shi, Y.; Xie, W. Z.; Zhou, H. M.; Int. J. Biochem. Cell Biol. 2000, 32, 879.

73. Komersová, A.; Masopustová, M.; Komers, K.; St pánková, S.; Cegan, A.; Z. Naturforsch. C 2007, 62, 305.

74. Venkateswara, R. J.; Shilpanjali, D.; Kavitha, P.; Madhavendra, S. S.; Arch. Toxicol. 2003, 77, 227.

75. Vincenzini, M. T.; Favilli, F.; Terves, C.; Vanni, P.; Life Sci. 1982, 31, 463.

76. Gupta, B. N.; Mathur, A. K.; Agarwal, C.; Singh, A.; Bull. Environ. Contam. Toxicol. 1989, 42, 375.

77. Joseph, E. M.; Morel, F. M. M.; Price, N. M.; Can. J. Fish. Aq. Sci. 1995, 52, 353 .

78. Repetto, G.; Peso, A. D.; Repetto, M.; Ecotoxicol. Environ. Restoration 2000, $31,47$.

79. Rahman, M. F.; Mahboob, M.; Gover, P.; Bull. Environ. Contam. Toxicol 2004, 72, 38.

80. Walz, I.; Schwack, W.; J. Agric. Food Chem. 2007, 55, 10563.

81. Henczová, M.; Deér, A. K.; Komlósi, V.; Mink, J.; Anal. Bioanal. Chem. 2006, 385, 652 .

82. Skladal, P.; Fiala, M.; Krejci, J.; Int. J. Environ. Anal. Chem. 1996, 65, 139.

83. Mazzei, F.; Botre, F.; Botre, C.; Anal. Chim. Acta 1996, 336, 67.

84. Gill, T. S.; Tewari, H.; Pande, J.; Comp. Biochem. Physiol. C 1990, 97, 287.

85. Durrieu, C.; Tran-Minh, C.; Ecotoxicol. Environ. Saf. 2002, 51, 206.

86. Chouteau, C.; Dzyadevych, S.; Durrieu, C.; Chovelon, J. M.; Biosens. Bioelect. 2005, 21, 273.

87. El Demerdash, F. M.; Elagamy, E. I.; Int. J. Environ. Health Res. 1999, 9, 173.

88. Tang, J. X.; Siegfried, B. D.; Hoagland, K. D.; Tang, J. X.; Pest. Bioch. Physiol. 1998, 59, 155.

89. Zhou, X. W.; Chen, Q. X.; Chen, Z.; He, Z. Q.; Zhou, H. M.; Biochem. (Moscow) 2000, 65, 1424.
90. Youngs, H. L.; Sundaramoorthy, M.; Gold, M. H.; Eur. J. Biochem. 2000, 27, 1761.

91. Bennun, A.; Blum, J. J.; Biochim. Biophys. Acta 1966, 128, 106.

92. Giannaccini, G.; Betti, L.; Palego, L.; Chelli, B.; Gallo, A.; Pirone, A.; Fabiani, O.; Bertellotti, S.; Lucacchini, A.; Comp. Biochem. Physiol. C Toxicol. Pharmacol. 2004, 137, 197.

93. Al Ghais, S. M.; Ahmad, S.; Ali, B.; Ecotoxicol. Environ. Saf. 2000, 46, 258.

94. Silva Filho, M. V.; Oliveira, M. M.; Salles, J. B.; Bastos, V. L.; Cassano, V. P.; Bastos, J. C.; Toxicol. Lett. 2004, 153, 247.

95. Salles, J. B.; Cunha Bastos, V. L.; Silva Filho, M. V.; Machado, O. L.; Salles, C. M.; Giovanni de Simone, S.; Cunha Bastos, J.; Biochimie 2006, $88,59$.

96. Guner, S.; Colak, A.; J. Biochem. Toxicol. 1996, 11, 257.

97. Gonzalez, O.; Fernandez, J.; Martin, M.; Comp. Biochem. Physiol. C 1987, 86, 163.

98. Maycock, D. S.; Prenner, M. M.; Kheir, R.; Morris, S.; Callaghan, A.; Whitehouse, P.; Morritt, D.; Crane, M.; Water Res. 2003, 37, 4180.

99. Balint, T.; Ferenczy, J.; Katai, F.; Kiss, I.; Kraczer, L.; Kufesak, O.; Lang, G.; Polyhos, C.; Szabo, I.; Szegletes, T.; Nemcsok, J.; Ecotoxicol. Environ. Saf. 1997, 37, 17.

100. Vlahogianni, T.; Dassenakis, M.; Scoullos, M. J.; Valavanidis, A.; Mar. Pollut. Bull. 2007, 54, 1361.

101. Adham , K. J.; Hassan, I. F.; Taha, N.; Amin, T. H.; Environ. Monit. Assess. 1999 54, 107.

102. Collen, J.; Pinto, E.; Pedersen, M.; Colepicolo, P.; Arch. Environ. Contam. Toxicol. 2003, 45, 337.

103. Kong, F. X.; Chen, Y.; Bull. Environ. Contam. Toxicol. 1995, 55, 759.

104. Peterson, S. M.; Stauber, J. L.; Bull. Environ. Contam. Toxicol. 1996, 56, 750.

105. Franklin, N. M.; Adams, M. S.; Stauber, J. L.; Lim, R. P.; Arch. Environ. Contam. Toxicol. 2001, 40, 469.

106. Hampel, M.; Moreno-Garrido, I.; Sobrino, C.; Lubian, L. M.; Blasco, J.; Ecotoxicol. Environ. Saf. 2001, 48, 287.

107. Devriese, M.; Tsakaloudi, V.; Garbayo, I.; Leon, R.; Vilchez, C.; Vigara, J.; Plant Physiol. Biochem. 2001, 39, 443.

108. Shizhong, T.; Zan, L.; Jianhua, W.; Yongyuan, Z.; Chemosphere 1997, $35,2713$.

109. Kong, F. X.; Hu, W.; Liu, Y.; Environ. Exp. Bot. 1998, 40, 105.

110. Young, L. M.; Woung, S. H.; J. Appl. Phycol. 2003, 15, 13.

111. Dominguez, M. J.; Gutierrez, F.; Leon, R.; Vilchez, C.; Veja, J. M.; Vigara, J.; Plant Physiol. Biochem. 2003, 41, 828.

112. Hiraki, M.; Ohki, S.; Sato, Y.; Jablonkai, I.; Boeger, P.; Wakabayashi, K.; Pest. Biochem. Physiol. 2001, 70, 159.

113. Zbigniew, T.; Wojciech, P.; Ecotoxicol. Environ. Saf. 2006, 65, 323.

114. Saeed, A.; Tremori, E.; Manao, G.; Camici, G.; Cappugi, G.; Ramponi, G.; Physiol. Chem. Phys. Med. NMR 1990, 22, 81.

115. Roche, M. J.; Bull. Soc. Chem. Biol. 1931, 13, 841.

116. Neuman, H.; J. Biol. Chem. 1968, 243, 4671.

117. Chaimovich, H.; Nome, F.; Arch. Biochem. Biophys. 1970, 139, 9.

118. Moore, M. N.; Viarengo, A.; Donkin, P.; Hawkins, A. J.; Aquat. Toxicol. 2007, 84, 80 .

119. Broeg, K.; Westernhagen, H. V.; Zander, S.; Körting, W.; Koehler, A.; Mar. Pollut. Bull. 2005, 50, 495.

120. Sturve, J.; Berglund, A.; Balk, L.; Broeg, K.; Bohmert, B.; Massey, S.; Savva, D.; Parkkonen, E.; Stephensen, E.; Koehler, A.; Forlin, L.; Environ. Toxicol. Chem. 2005, 24, 1951.

121. Bottini, N.; Gloria-Bottini, F.; Borgiani, P.; Antonacci, E.; Lucarelli, P.; Bottini, E.; Metab. Clin. Exp. 2004, 53, 995.

122. Zhang, W.; Gruszewski, H. A.; Chevone, B. I., Nessler, C. L.; Pl. Physiol. 2008, 146, 431.

123. El Hissy, F. T.; El Nagdy, M. A.; El Sharouny, H. M.; Abd Elaah, G. A.; Folia Microbiol. 1995, 40, 341. 
124. Nicolau, A.; Mota, M.; Lima, N.; Ecotoxicol. Environ. Saf. 2004, 57, 129.

125. Nakazato, H.; Okamoto, T.; Ishikawa, K.; Okuyama, H.; Plant Physiol. Biochem. 1997, 35, 437.

126. Khangarot, B. S.; Rathore, R. S.; Bull. Environ. Contam. Toxicol. 2003, 70, 112.

127. Reddy, M. S.; Rao, K. V. R.; Biochem. Int. 1990, 22, 1033.

128. Geraldine, P.; Bhavan, P. S.; Kaliamurthy, J.; Zayapragassarazan, Z.; J. Environ. Biol. 1999, 20,141.

129. Bhavan, P. S.; Geraldine, P.; J. Environ. Biol. 2004, 25, 213.

130. De And, A.; Sur, R. K.; Environ. Ecol. 1990, 8, 107.

131. Teh, S. J.; Clark, S. L.; Brown, C. L.; Luoma, S. N.; Hinton, D. E.; Biomarkers 1999, 4, 497.

132. Giamberini, L.; Pihan, J. C.; Diseases Aquat. Organ. 1997, 28, 221.

133. Jing, G.; Li, Y.; Xie, L.; Zhang, R.; Comp. Biochem. Physiol. C Toxicol. Pharmacol. 2006, 144, 184.

134. Radwan, M. A.; El Wakil, H. B.; Osman, K. A.; J. Environ. Sci. Health B 1992, 27, 759 .

135. Bream, A. S.; Commun. Agric. Appl. Biol. Sci. 2003, 68, 291.

136. Strmac, M.; Braunbeck, T.; Toxicol. In Vitro 2000, 14, 361.

137. Vijayavel, K.; Balasubramanian, M. P.; Ecotoxicol. Environ. Saf. 2007, $66,154$.

138. Sharma, R. M.; Bull. Environ. Contam. Toxicol. 1990, 44, 443.

139. Das, B. K.; Mukherjee, S. C.; Toxicol. Lett. 2000, 114, 11.

140. Sheela, M.; Muniandy, S.; Environ. Ecol. 1992, 10, 220.

141. Kumar, K.; Ansari, B. A.; Ecotoxicol. Environ. Saf. 1986, 12, 199.

142. Agrahar, S.; Pandey, K. C.; Gopal, K.; Pest. Biochem. Physiol. 2007, 88, 268.

143. Barse, A.; Chakrabarti, T.; Ghosh, T. K.; Pal, A. K.; Jadhao, S. B.; Pest. Biochem. Physiol. 2006, 86, 172.

144. Fahraeus, V. R. G. E.; Spurrell, D. R.; Ecotoxicol. Environ. Saf. 2000, $46,289$.
145. Misra, V.; Kumar, V., Pandey, S. D.; Viswanathan, P. N.; Arch. Environ. Contam. Toxicol. 1991, 21, 514.

146. Mohan, D.; Verma, S. R.; Toxicol. Lett. 1981, 8, 171.

147. Versteeg, D. J.; Giesy, J. P.; Ecotoxicol. Environ. Saf. 1986, 11, 31.

148. Verma, S. R.; Tonk, I. P.; Chand, R.; Clin. Physiol. Biochem. 1985, 3, 199.

149. Abd Elaah, G. A.; El Nagdy, M. A.; African J. Mycol. Biotechnol. 1999, 7,7 .

150. Cooper, A.; Bowen, I. D.; Lloyd, D.; J. Cell. Sci. 1974, 15, 605

151. Braten, T.; J. Cell. Sci. 1975, 17, 647.

152. Domozych, D. S.; Protoplasma 1989, 149, 108.

153. Kruskopf, M. M.; Du Plessis, S.; Hydrobiologia 2004, 513, 59.

154. Tsekos, I.; Schnepf, E.; Pl. Syst. Evol. 1991, 176, 35.

155. Tornqvist, L.; Environ. Exp. Bot. 1989, 29, 457.

156. Sommer, J. R.; Blum, J. J.; J. Cell. Biol. 1965, 24, 235.

157. Vrba, J.; Komarkova, J.; Vyhnalek, V.; Water Sci. Technol. 1993, $28,15$.

158. Bachir, F.; Plant Sci. 1996, 119, 93.

159. Bachir, F.; Loppes, R.; FEMS Microbiol. Lett. 1997, 149, 195.

160. Knutsen, G.; Biochim. Biophys. Acta 1968, 161, 205.

161. Loppes, R.; Matagne, R. F.; Genetics 1973, 75, 604.

162. Matagne, R. F.; Loppes, R.; Deltour, R.; J. Bacteriol. 1976, 126, 937.

163. Patni, N. J.; Aaronson, S.; J. Gen. Microbiol. 1974, 83, 9.

164. Lien, T.; Knutsen, G.; Physiol. Plant 1973, 28, 298.

165. Nagy, A. H.; Erdos, G.; Beliaeva, N. N.; Gyurjan, I..; Mol. Gen. Genet. 1981, 184, 314

166. Yamamoto, M.; Phytochemistry 1972, 11, 2451.

167. Schmitter, R. E.; Jurkiewicz, A. J.; J. Cell. Sci. 1981, 51, 15.

168. Lee, T. M.; Huang, Y. L.; Chen, M. H.; Phycologia 2005, 44, 620.

169. Rai, L. C.; Rai, P. K.; Mallick, N.; Environ. Exp. Bot. 1996, 36, 99.

170. Brasil, Ministério do Meio Ambiente, Conselho Nacional do Meio Ambiente-CONAMA; Resolução $n^{0}$ 357, de 17 de março de 2005, Diário Oficial da União, Brasília, 18 de março de 2005. 